\title{
Hypophysitis secondary to nivolumab and pembrolizumab is a clinical entity distinct from ipilimumab-associated hypophysitis
}

\author{
Alexander Faje ${ }^{1}$, Kerry Reynolds², Leyre Zubiri², Donald Lawrence ${ }^{3}$, Justine V Cohen ${ }^{3}$, Ryan J Sullivan ${ }^{3}$, \\ Lisa Nachtigall' ${ }^{1}$ and Nicholas Tritos ${ }^{1}$ \\ ${ }^{1}$ Neuroendocrine Unit, Massachusetts General Hospital and Harvard Medical School, Boston, Massachusetts, USA, \\ ${ }^{2}$ Division of Oncology, and ${ }^{3}$ Center for Melanoma, Massachusetts General Hospital Cancer Center, Boston, \\ Massachusetts, USA \\ Correspondence \\ should be addressed \\ to A Faje \\ Email \\ afaje@partners.org
}

\begin{abstract}
Objective: Little has been published describing hypophysitis after nivolumab or pembrolizumab treatment. We aimed to (i) assess the risk of hypophysitis following nivolumab or pembrolizumab treatment, (ii) characterize the clinical presentation and outcomes in these patients and (iii) compare these patients to hypophysitis following ipilimumab and ipilimumab plus nivolumab (combo). We hypothesized that headaches, pituitary enlargement on MRI and multiple anterior pituitary hormone deficiencies would occur less often in the nivolumab/pembrolizumab group versus ipilimumab or combo hypophysitis patients.

Design and methods: We conducted a multi-center retrospective review utilizing the Research Patient Database registry to evaluate individuals diagnosed with hypophysitis following treatment with nivolumab/pembrolizumab $(n=22)$, ipilimumab $(n=64)$ and combo $(n=20)$. Encounter notes, radiologic imaging and laboratory results for these patients were comprehensively reviewed.

Results: Hypophysitis was rare following treatment with nivolumab/pembrolizumab $(0.5 \%, 17 / 3522)$ compared to ipilimumab (13.6\%, 34/250), $P<0.0001$. Hypophysitis was diagnosed later in nivolumab/pembrolizumab (median: 25.8 weeks, interquartile range (IR): 18.4-44.0) compared to ipilimumab (9.3, IR: 7.2-11.1) or combo patients (12.5, IR: 7.4-18.6), $P<0.0001$ for both. Headache and pituitary enlargement occurred less commonly in nivolumab/ pemrolizumab patients ( $23 \%$ and $5 / 18$, respectively) compared to ipilimumab $(75 \%, 60 / 61)$ and combo $(75 \%, 16 / 17)$ treatment groups $(P<0.0001$ versus ipilimumab and $P=0.001$ versus combo for headache and $P<0.0001$ for both for enlargement).

Conclusions: This study represents the first comprehensive cohort analysis of nivolumab or pembrolizumab-associated hypophysitis in a large patient group. Hypophysitis occurs rarely with these medications, and these patients have a distinct phenotype compared to hypophysitis after treatment with ipilimumab or ipilimumab plus nivolumab.
\end{abstract}

\section{Introduction}

Seven immune checkpoint inhibitors (CPIs) are currently approved by the US Food and Drug Administration (FDA) (ipilimumab, nivolumab, pembrolizumab, cemiplimab, atezolizumab, avelumab and durvalumab). FDA-approved treatment indications for CPIs have expanded greatly in recent years and now include at least 15 different cancer types. The number of patients treated with these medications has increased in parallel; approximately 2000 new treatment initiations have taken place at our institution alone during the past two and a half years.

Published by Bioscientifica Ltd. 
Ipilimumab-associated hypophysitis is a welldescribed immune-related adverse event (irAE) (1, 2, 3). In comparison, based on data derived from prospective CPI trials, hypophysitis appears to occur more rarely following treatment with agents targeting programmed cell death 1 (PD-1) $(4,5,6)$. Limitations in the existing common terminology criteria for adverse events (CTCAE), which contains multiple imprecise and overlapping endocrine categories, likely impact the accuracy of endocrine irAE reports in these trials. Clinical descriptions of patients diagnosed with hypophysitis following treatment with anti-PD-1 medications are very sparse and consist of case reports and letters with little or no longitudinal information $(7,8,9,10,11,12,13,14,15,16,17,18,19$, $20,21,22,23,24,25,26,27,28,29)$. To our knowledge, no cohort study examining such patients has been published.

We performed a comprehensive retrospective review of patients who developed hypophysitis following treatment with nivolumab or pembrolizumab monotherapy at Massachusetts General Hospital (MGH) and Brigham and Women's Hospital (BWH)/Dana-Farber Cancer Institute (DFCI). We compared these patients to those who developed hypophysitis following treatment with ipilimumab monotherapy and combination therapy with ipilimumab plus concurrent nivolumab. Based on our clinical observations and experience with patients receiving CPIs, we anticipated that hypophysitis secondary to anti-PD-1 monotherapy would have a distinct clinical phenotype compared to cases associated with ipilimumab alone or in combination with nivolumab. Specifically, we hypothesized that headache, pituitary enlargement on MRI and multiple pituitary hormone deficiencies would occur less frequently in the anti-PD-1 monotherapy hypophysitis patients compared to the other two groups.

\section{Patients and methods}

The study was performed at the MGH, Boston, MA, USA, approved by the Institutional Review Board of Partners Healthcare, Boston, and conforms to the STROBE Initiative recommendations (30). The Research Patient Database registry (RPDR) was utilized to search patient medical records. The RPDR is an electronic repository containing data from greater than 7 million patients, including more than 2.6 billion coded records and 130 million clinical notes, in the Partners healthcare system dating to 1993.

We retrospectively identified all melanoma patients at Partners-affiliated hospitals who developed hypophysitis following treatment with ipilimumab monotherapy
( $n=64)$ or combination ipilimumab plus nivolumab therapy ( $n=20)$ thru August 1, 2017 as previously described (2). As previously noted, our group independently maintained a list of all melanoma patients treated with ipilimumab monotherapy at MGH thru August 1, 2017 $(n=250)$.

Our group has also maintained an active list of patients with any malignancy treated with pembrolizumab or nivolumab monotherapy at MGH who developed hypophysitis. The RPDR was utilized to supplement this list and identify all other patients who developed hypophysitis following treatment with nivolumab or pembrolizumab monotherapy at MGH and BWH/DFCI. Inpatient and outpatient clinical notes through September 1, 2018 were searched for the following text combination: (1) 'nivolumab' OR 'pembrolizumab' plus (2) 'hypophysitis' OR 'hypopituitarism' OR 'adrenal insufficiency'. The search results were also restricted to patients who had received a formal endocrinology consultation. The database query yielded 266 patients. Encounter notes, radiologic imaging and laboratory results for these patients were comprehensively reviewed. In total, we identified 22 patients who developed hypophysitis after treatment with nivolumab or pembrolizumab monotherapy. Three additional probable cases of hypophysitis secondary to nivolumab or pembrolizumab were identified. Following consensus review by the authors, these patients were not included in the formal data analyses of the manuscript. A database of all patients receiving CPIs at Partners-affiliated hospitals from April 2, 2016 onwards was reviewed and identified 3522 new treatment initiations of nivolumab or pembrolizumab monotherapy in 3479 patients with any cancer type through September 1, 2018 at MGH or BWH/DFCI.

Previously published cases of hypophysitis associated with anti-PD-1 agents were identified by searching PubMed for all English-language articles through December 1, 2018 utilizing the terms 'nivolumab hypophysitis', 'pembrolizumab hypophysitis', 'nivolumab adrenal insufficiency', 'pembrolizumab adrenal insufficiency', 'nivolumab hypopituitarism', 'pembrolizumab hypopituitarism', 'nivolumab ACTH', 'pembrolizumab ACTH'. Twenty-three case reports and letters were identified (7, $8,9,10,11,12,13,14,15,16,17,18,19,20,21,22,23$, $24,25,26,27,28,29)$. The full text of one manuscript could not be obtained (7). The remaining 22 publications described 28 patient cases.

Clinical diagnoses of hypophysitis were based on the presence of new hypopituitarism and/or radiographic pituitary enlargement following treatment with CPIs 
in the absence of an alternative etiology (including assessment of prior exogenous glucocorticoid exposure). Magnetic resonance imaging (MRI) results were included if performed within 1 month of the diagnosis of hypophysitis. This time period was selected due to our group's observation that pituitary gland enlargement resolves in approximately half of patients with ipilimumabassociated hypophysitis within a month (1). All MRIs were reviewed by a neuroradiologist and neuroendocrinologist. Hypopituitarism was defined as previously described (2). Similar criteria were applied to our review of previously published cases. If laboratory values were not detailed in those cases, the respective parameters were considered to be unavailable. GH stimulation testing was not routinely performed in our cohort but was frequently performed in the prior published cases and included for the analysis of growth hormone deficiency. The timing of hormonal testing varied according to the discretion of their physicians.

JMP (version 14, SAS Institute) was utilized for statistical analysis. Values are shown as means \pm s.E.M., unless otherwise noted. $P$ values $<0.05$ were considered statistically significant. Data normality was assessed by the Wilk-Shapiro test. One-way ANOVA was performed for initial group comparisons with continuous outcomes when data were normally distributed, and the KruskalWallis was utilized when data were not normally distributed (followed by post hoc testing with the Wilcoxon rank-sum test for individual group comparisons). The chisquared test was applied for initial and post hoc testing for group comparisons with binary measures.

\section{Results}

Twenty-two patients were diagnosed with hypophysitis following treatment with nivolumab $(n=7)$ or pembrolizumab $(n=15)$ monotherapy from any date through September 1, 2018. The calculated risk of developing hypophysitis was $0.50 \%$ (17 cases among 3522 new treatment initiations in 3479 patients with nivolumab or pembrolizumab monotherapy specifically between April 2, 2016 and September 1, 2018). The risks of hypophysitis individually for nivolumab $(0.03 \%)$ and pembrolizumab $(0.06 \%)$ did not differ significantly $(P=0.27)$. The low rate for nivolumab and pembrolizumab contrasted with a much higher incidence of $13.6 \%(34 / 250)$ following treatment with ipilimumab monotherapy $(P<0.0001)$. Our available datasets did not include the total number of patients treated with combination therapy, and we were therefore unable to calculate the risk of hypophysitis in this group. In the anti-PD-1 monotherapy hypophysitis group, underlying malignancies included melanoma $(n=12)$, lung cancer $(n=4$; one small cell and three non-small cell), ovarian cancer $(n=3)$, and colorectal, breast and squamous cell cancer of the oropharynx ( $n=1$ for each).

Age and gender distribution did not differ between patients with hypophysitis secondary to anti-PD-1, ipilimumab or combination therapy (Table 1). We have previously reported a lack of gender predilection among patients with ipilimumab-induced hypophysitis (1). Dataset limitations precluded gender ratio calculations for patients with anti-PD-1 and combination therapy-induced hypophysitis versus patients without. As previously noted hypophysitis after treatment with ipilimumab often occurs within a relatively narrow timeframe. Hypophysitis occurred later and more variably in the anti-PD-1 group, ranging from 11 to 79 weeks (4-33 cycles) following treatment initiation. The time range for combination therapy patients appeared to be intermediate between the two other groups but with a greater similarity to ipilimumab monotherapy patients (Fig. 1 and Table 1).

Pituitary gland enlargement was observed in nearly all patients with hypophysitis following treatment with ipilimumab, whether it was administered by itself (98\%) or in combination with nivolumab (94\%), but only in a minority of patients who were treated with nivolumab or pembrolizumab alone (28\%) (Table 1). Gland enlargement, when present, was mild and did not cause optic chiasm compression in any patient of the three groups. The presenting symptoms of hypophysitis patients treated with anti-PD-1 monotherapy differed from the other groups. Specifically, headache was a more frequent symptom in patients treated with ipilimumab or combination therapy compared to anti-PD-1 monotherapy patients, whose primary symptoms were fatigue, loss of appetite and myalgias/arthralgias. Among the five anti-PD-1 hypophysitis patients who did note headache, $2 / 4$ had pituitary gland enlargement on MRI (1 patient did not have imaging performed at the time of diagnosis). Four of these five patients had hyponatremia at the time of diagnosis, including the two patients with normal pituitary MRIs. Hyponatremia was present at the time of diagnosis in a substantial proportion of all three groups (Table 1).

The pattern of anterior pituitary hormone deficiencies at the time of diagnosis also differed in patients receiving anti-PD-1 monotherapy. These patients often only had isolated central adrenal insufficiency (18/22). Deficiencies 
Table 1 Immune checkpoint inhibitor-associated hypophysitis, clinical presentation and outcomes. Data are presented as mean \pm S.E.M.or as median (IR).

\begin{tabular}{|c|c|c|c|c|}
\hline & Anti-PD-1 $(n=22)$ & Ipilimumab $(n=64)$ & Combination therapy $(n=20)$ & $P$ value \\
\hline Age (years) & $61.5 \pm 1.6$ & $65.8 \pm 1.5$ & $60.7 \pm 2.6$ & 0.12 \\
\hline Gender $(m, f)$ & 12,10 & 48,16 & 11,9 & 0.10 \\
\hline $\begin{array}{l}\text { Time to diagnosis after treatment } \\
\text { initiation (weeks) }\end{array}$ & $25.8(18.4-44.0)$ & $9.3(7.2-11.1)$ & $12.5(7.4-18.6)$ & $<0.0001^{* \neq}, 0.02^{\dagger}$ \\
\hline Treatment cycles at diagnosis & $10(7-13)$ & $3(3-4)$ & $4(3-4)$ & $<0.0001^{*}, \neq, 0.10^{\dagger}$ \\
\hline Treatment cycles, total & $18(13-25)$ & $4(3-4)$ & $4(4-4)$ & $<0.0001^{*, \mp}, 0.05^{\dagger}$ \\
\hline Pituitary enlargement on MRI & $5 / 18$ & $60 / 61$ & $16 / 17$ & $<0.0001^{*, \neq}, 0.37^{\dagger}$ \\
\hline Hyponatremia at diagnosis & $11 / 22$ & $29 / 64$ & $6 / 19$ & 0.45 \\
\hline $\begin{array}{l}\text { Cranial radiotherapy for } \\
\text { metastatic disease at any time }\end{array}$ & $3 / 22$ & $28 / 64$ & $8 / 20$ & $0.01^{*}, 0.77^{\dagger}, 0.05^{\ddagger}$ \\
\hline \multicolumn{5}{|l|}{ Presenting symptoms $(n, \%)$} \\
\hline Headache & $5(23)$ & $48(75)$ & $15(75)$ & $<0.0001^{*}, 1.00^{\dagger}, 0.001^{\ddagger}$ \\
\hline Fatigue & $21(95)$ & $37(58)$ & $12(60)$ & $0.0003^{*}, 0.86^{\dagger}, 0.003^{\ddagger}$ \\
\hline Nausea/appetite loss & $15(68)$ & $28(44)$ & $7(35)$ & $0.05^{*}, 0.49^{\dagger}, 0.03^{\ddagger}$ \\
\hline Dizziness & $3(14)$ & $7(11)$ & $2(10)$ & 0.92 \\
\hline Altered mental status & $1(5)$ & $8(13)$ & $1(5)$ & 0.38 \\
\hline Myalgias/arthralgias & $8(36)$ & $4(6)$ & $2(10)$ & $0.001^{*}, 0.58^{\dagger}, 0.04^{\ddagger}$ \\
\hline Low blood pressure & $4(18)$ & $3(5)$ & $1(5)$ & 0.16 \\
\hline \multicolumn{5}{|l|}{ Hypopituitarism at diagnosis } \\
\hline Any & $21 / 22^{\S}$ & $61 / 64 \|$ & $17 / 20^{9}$ & 0.32 \\
\hline Hypothyroidism & $3 / 22$ & $52 / 64$ & $12 / 20$ & $<0.0001^{*}, 0.06^{\dagger}, 0.001^{\ddagger}$ \\
\hline Hypoadrenalism & $21 / 21$ & $32 / 58$ & $10 / 16$ & $<0.0001^{*}, 0.60^{\dagger}, 0.001^{\ddagger}$ \\
\hline Hypogonadism & $1 / 13$ & $45 / 51$ & $5 / 9$ & $<0.0001^{\star}, 0.03^{\dagger}, 0.01^{\ddagger}$ \\
\hline GHD (low IGF-1) & $0 / 8$ & $3 / 24$ & $0 / 4$ & 0.28 \\
\hline Prolactin (elevated, low) & $8 / 13 ; 0 / 13$ & $3 / 36 ; 23 / 36$ & $2 / 8 ; 1 / 8$ & $\begin{array}{l}0.0002^{*} ; 0.22^{\dagger}, 0.10^{\ddagger} \\
\quad<0.0001^{*}, 0.01^{\dagger}, 0.16^{\ddagger}\end{array}$ \\
\hline Diabetes insipidus & $0 / 22$ & $0 / 64$ & $0 / 20$ & NA \\
\hline \multicolumn{5}{|l|}{ Hypopituitarism at most recent visit } \\
\hline Any & $17 / 20$ & $62 / 63$ & $19 / 20$ & 0.09 \\
\hline Hypothyroidism & $1 / 16$ & $36 / 61$ & $5 / 20$ & $<0.0001^{*}, 0.01^{\dagger}, 0.12^{\ddagger}$ \\
\hline Hypoadrenalism & $16 / 16$ & $60 / 62$ & $19 / 20$ & 0.55 \\
\hline Hypogonadism & $0 / 8$ & $24 / 44$ & $2 / 6$ & $0.001^{*}, 0.33^{\dagger}, 0.05^{\ddagger}$ \\
\hline GHD (low IGF-1) & $0 / 4$ & $1 / 12$ & $0 / 1$ & 0.70 \\
\hline Prolactin (elevated, low) & $1 / 4,0 / 4$ & $1 / 11,3 / 11$ & $1 / 2,1 / 2$ & $0.39 ; 0.24$ \\
\hline Diabetes insipidus & $0 / 22$ & $0 / 64$ & $0 / 20$ & NA \\
\hline
\end{tabular}

${ }^{*} P$ value between Anti-PD-1 and Ipilimumab; ${ }^{\dagger} P$ value between ipilimumab and combination therapy; ${ }^{\ddagger} P$ value between anti-PD- 1 and combination therapy; ${ }^{5}$ anterior pituitary biochemical assessment was limited to the thyroid axis at the time of diagnosis in one patient; " anterior pituitary biochemical assessment was limited to the thyroid axis and adrenal at the time of diagnosis in three patients; "anterior pituitary biochemical assessment was limited to the thyroid axis at the time of diagnosis in one patient and the thyroid and adrenal axis in two patients.

f, female; GHD, growth hormone deficiency; m, male.

in other pituitary axes were observed more often in the ipilimumab monotherapy and combination therapy groups. Diabetes insipidus did not occur in any patient among the three groups. In all three groups, central adrenal insufficiency was nearly always persistent (Table 1).

The biochemical severity of central adrenal insufficiency in the anti-PD-1 monotherapy patients was notable. The peak cortisol value during an intravenous $250 \mu \mathrm{g}$ cosyntropin stimulation test did not exceed $3.7 \mu \mathrm{g} / \mathrm{dL}$ in 8 of 13 patients. Early morning cortisol values were undetectable $(<0.5 \mu \mathrm{g} / \mathrm{dL})$ in 5 of 6 other patients who did not undergo cosyntropin stimulation testing.
There was no case of recurrent hypophysitis in any group. A majority of the ipilimumab and combination therapy patients received additional doses of CPIs (31). Fifteen of the 22 anti-PD-1 hypophysitis patients received additional doses of CPIs. Five patients have not required any further systemic treatment after the diagnosis of hypophysitis. Patients in the anti-PD-1 group were typically treated with physiologic glucocorticoid doses or briefly received mildly supraphysiologic doses. Peak glucocorticoid doses exceeded $10 \mathrm{mg}$ prednisone equivalent per day in only 3 out of 22 patients in this group. Glucocorticoid dosing and survival were analyzed 

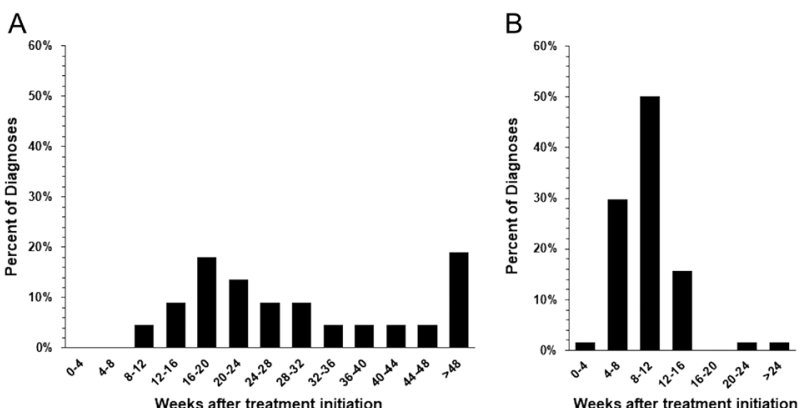

in detail previously for ipilimumab hypophysitis patients (2). Detailed survival analyses could not be performed in the anti-PD-1 group since these patients had multiple different malignancies, but it was notable that all patients were alive at the time of the study, with a mean follow-up of $20.8 \pm 1.9$ months.

Primary thyroid dysfunction is the most common endocrine irAE for patients treated with anti-PD-1 agents $(4,32,33)$. Seven $(32 \%)$ patients in the anti-PD-1 group and 7 (35\%) in the combination therapy group were also diagnosed with primary thyroid dysfunction following CPI treatment. Thyroid irAEs were diagnosed $32.1 \pm 6.0$ weeks after treatment initiation in the anti-PD-1 group and $14.9 \pm 2.4$ weeks in the combination therapy group $(P=0.03)$. Three diagnoses of thyroid irAEs occurred

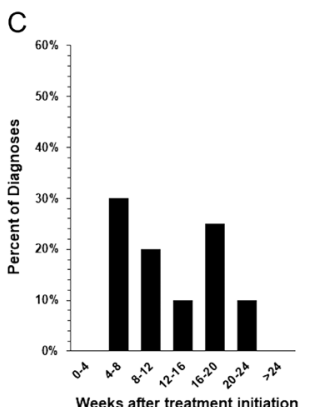

\begin{abstract}
Figure 1
Time (weeks) from the onset of therapy until the diagnosis of hypophysitis for patients treated with anti-PD-1 monotherapy (Panel A), ipilimumab monotherapy (Panel B) and ipilimumab plus concurrent nivolumab (Panel C).
\end{abstract}

before hypophysitis in the anti-PD-1 group, and four took place afterwards. In the combination therapy group, all seven patients were diagnosed with primary thyroid dysfunction prior to hypophysitis, and three occurred less than 4 weeks apart from the development of hypophysitis.

The characteristics of the three additional anti-PD-1 patients with probable but not definite diagnoses of hypophysitis were similar to the overall group. A sensitivity analysis that included these patients did not change any of the findings reported above.

To our knowledge, no prior cohort study of hypophysitis associated with anti-PD-1 agents has been published. A literature search identified 28 patient cases (27 following treatment with nivolumab and 1 after therapy with pembrolizumab). The data extracted from

Table 2 Cohort comparison with previously published cases. Data are presented as mean \pm S.E.M.or as median (IR).

\begin{tabular}{l}
\hline Age (years) \\
Gender (m, f) \\
Nivolumab, pembrolizumab $(n)$ \\
Time to diagnosis after treatment initiation (weeks) \\
Treatment cycles at time of diagnosis \\
Pituitary enlargement on MRI \\
Hyponatremia at diagnosis \\
Presenting symptoms ( $(\%))$ \\
Headache \\
Fatigue \\
Nausea/appetite loss \\
Dizziness \\
Altered mental status \\
Myalgias/arthralgias \\
Low blood pressure \\
Hypopituitarism at diagnosis \\
Any \\
Hypothyroidism \\
Hypoadrenalism \\
Hypogonadism \\
Growth hormone deficiency \\
Prolactin (elevated, low) \\
Diabetes insipidus
\end{tabular}

\begin{tabular}{c}
\hline Present cohort $(n=22)$ \\
\hline $61.5 \pm 1.6$ \\
12,10 \\
7,15 \\
$25.8(18.4-44.0)$ \\
$10(7-13)$ \\
$5 / 18$ \\
$11 / 22$ \\
$5(23)$ \\
$21(95)$ \\
$15(68)$ \\
$3(14)$ \\
$1(5)$ \\
$8(36)$ \\
$4(18)$ \\
$21 / 22$ \\
$3 / 22$ \\
$21 / 21$ \\
$1 / 13$ \\
$0 / 8$ \\
$8 / 13 ; 0 / 13$ \\
$0 / 22$
\end{tabular}

\begin{tabular}{c}
\hline Prior cases $(n=28)^{*}$ \\
\hline $63.8 \pm 2.2$ \\
22,6 \\
27,1 \\
10 weeks to 15 months after 24 cycles $^{\dagger}$ \\
$2-28^{\dagger}$ \\
$2 / 24$ \\
$12 / 21$ \\
$0(0)$ \\
$23(82)$ \\
$17(61)$ \\
$3(11)$ \\
$1(4)$ \\
$5(18)$ \\
$7(25)$ \\
$27 / 28$ \\
$0 / 19$ \\
$27 / 28$ \\
$0 / 10$ \\
$2 / 14$ \\
$5 / 17,0 / 17$ \\
$0 / 28$
\end{tabular}

*Cases were compiled from references $(7,8,9,10,11,12,13,14,15,16,17,18,19,20,21,22,23,24,25,26,27,28,29) ;{ }^{\dagger}$ Timing was reported in a variable manner and therefore formal statistical analysis could not be performed. $\mathrm{m}$, male; f, female. 


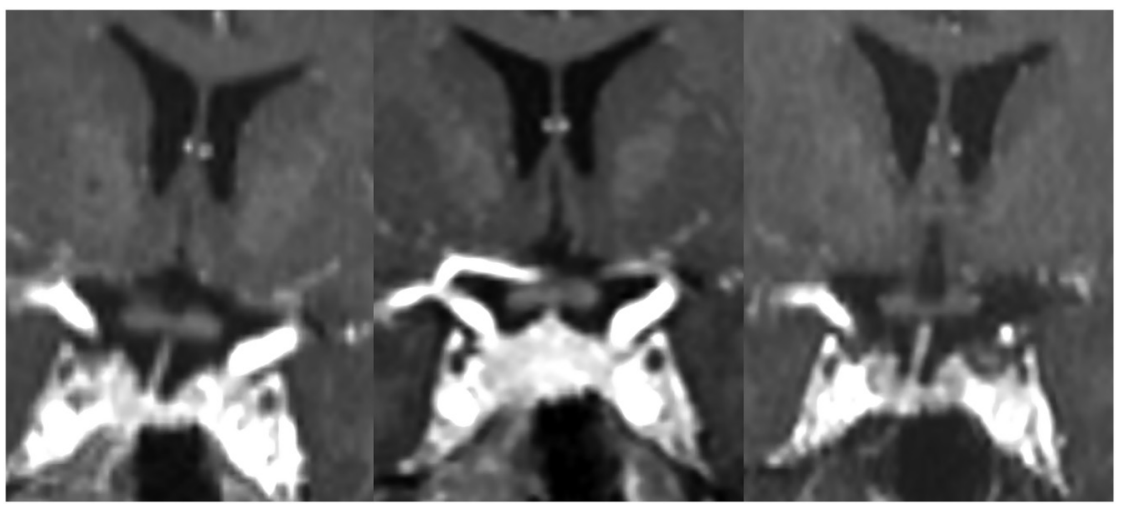

\section{Figure 2}

MRI images of the pituitary gland 3 days prior to pembrolizumab initiation, 3 months after starting pembrolizumab and 3 months after hypophysitis occurred. Pituitary gland enlargement was not recognized by the interpreting radiologist during admission for fatigue/malaise and failure to thrive, symptoms which promptly resolved with glucocorticoid treatment.

these reports were summarized in Table 2 . The aggregated findings closely mirrored our data.

\section{Discussion}

This is the first cohort study and longitudinal analysis of patients with hypophysitis secondary to treatment with anti-PD-1 medications, identified among a large population at risk, as well as the first formal comparison of hypophysitis following treatment with anti-PD-1 monotherapy, ipilimumab monotherapy and ipilimumab plus nivolumab combination therapy. Existing published data for hypophysitis associated with PD-1 blockade are sparse and limited to case reports and letters with little longitudinal information. Our analysis helps establish the clinical phenotype of these patients and distinguishes them from hypophysitis occurring after treatment with ipilimumab alone or in combination with nivolumab.

The risk of developing hypophysitis following anti-PD-1 monotherapy was more than an order of magnitude lower compared to treatment with ipilimumab. To our knowledge, the number of anti-PD-1treated patients in this study is the largest group reported to date and is double the size examined in a recent metaanalysis of endocrine irAEs (4). Our study constitutes perhaps the most reliable assessment of hypophysitis risk following treatment with anti-PD-1 monotherapy. The meta-analysis by Barroso-Sousa et al. was derived from prospective oncologic studies which 'clearly reported an endocrine AE in their safety data'. They reported a similar rate of hypophysitis for anti-PD-1 monotherapy patients but a much lower value for ipilimumab (4) compared to this study, more recent oncologic investigations (6) and other endocrine-based investigations (1). This discordance may result from certain inadequacies of the current (version 5.0) and past CTCAE endocrine categories.
For example, current potentially pituitary-related CTCAE categories include the following: hypophysitis, hypopituitarism, adrenal insufficiency, testosterone deficiency, hypothyroidism, blood corticotrophin level decreased, blood gonadotropin abnormal. The presence or absence of other medication-related effects (such as exogenous glucocorticoids) is left unspecified and precise terminology (e.g., primary versus central) is lacking. Typically, most prospective oncology studies have simultaneously reported multiple of the potentially overlapping categories noted above. For example, the phase three trial for ipilimumab in melanoma by Hodi et al., reported events for hypophysitis, hypopituitarism and adrenal insufficiency separately (34). Postow et al. listed separate categories for the following: thyroid disorder, blood TSH decreased, hypophysitis, adrenal insufficiency, hypothyroidism and hyperthyroidism (35). Calculating accurate estimates of risk from such studies is challenging. It remains somewhat unclear if the risk of hypophysitis following combination therapy with ipilimumab plus nivolumab is greater than ipilimumab alone. As previously noted we were unable to calculate the risk for combination therapy patients from our available dataset.

In patients treated with anti-PD-1 monotherapy, hypophysitis was diagnosed at variable and typically later time points than those receiving ipilimumab monotherapy or combination therapy. Given the frequent lack of localizing symptoms (such as severe headache) or pituitary enlargement on MRI in the majority of anti-PD-1 monotherapy hypophysitis patients and the nonspecific nature of their primary presenting symptoms, delayed clinical recognition and diagnosis (or even underdiagnosis) for these patients is possible. It is also possible that such a delay in diagnosis may contribute to negative MRI findings in these patients, since pituitary gland enlargement often resolves rapidly in patients with 
hypophysitis secondary to ipilimumab (2). Even when pituitary enlargement occurs (Fig. 2), it may not always be recognized by the treatment team. In that particular case, the patient had multiple admissions for fatigue, failure to thrive and low-grade fevers that promptly responded to glucocorticoid treatment and recurred following discontinuation. The patient was later diagnosed with an inflammatory arthropathy and treated with pharmacologic doses of glucocorticoids for a couple of months followed by physiologic replacement dosages. She was unable to tolerate physiologic glucocorticoid discontinuation due to fatigue and weakness and had an undetectable serum cortisol following a $250 \mu \mathrm{g}$ cosyntropin stimulation test a year later.

Potential diagnostic delay does not appear to have a significant contribution to the distinct clinical phenotype observed in anti-PD-1 hypophysitis patients. Symptomatically, radiographically and biochemically, hypophysitis appears to have a subtler presentation following treatment with anti-PD-1 agents compared to ipilimumab. The two groups do retain some notable similarities. Hyponatremia occurs in a similar proportion of patients, and central adrenal insufficiency is nearly always persistent in both groups. In some aspects, patients treated with combination therapy appeared to be a partial hybrid of the monotherapy groups (for example, the timecourse of hypophysitis), but characteristics of the combination therapy patients overall aligned much more closely with ipilimumab monotherapy hypophysitis patients. Considering that hypophysitis is much more common following treatment with ipilimumab, this medication is likely the more dominant contributing factor in the presentation of combination therapyinduced hypophysitis.

The distinct clinical phenotype of anti-PD-1 versus ipilimumab-induced hypophysitis lends indirect support for the proposed mechanism of ipilimumab-mediated hypophysitis, namely, direct targeting of anterior pituitary cells by the monoclonal antibody. Nivolumab and pembrolizumab are IgG4-based monoclonal antibodies and do not effectively activate the classical complement pathway or antibody-dependent cellmediated cytotoxicity $(1,36,37)$. Precise mechanistic details for anti-PD-1-associated hypophysitis are currently unknown. In our study, the risk of hypophysitis was approximately 27-fold higher following treatment with ipilimumab compared to anti-PD-1 agents. Multiple toxicities, such as gastrointestinal complications, have been observed at higher frequencies with ipilimumab compared to anti-PD-1 therapy (38), but no irAE has been described with a difference of such magnitude as we have observed with hypophysitis. This finding is noteworthy considering the extreme rarity of primary hypophysitis versus other endocrinopathies, such as autoimmune thyroid disease or autoimmune diabetes mellitus, which can manifest as a CPI irAE.

Hypophysitis appears to be associated with improved survival after treatment with ipilimumab monotherapy in patients with melanoma (2). Due to the variety of cancer diagnoses in our cohort, we could not perform a meaningful survival analysis in anti-PD-1 hypophysitis patients. However, a diagnosis of hypophysitis did not appear to portend a poorer outcome in patients treated with anti-PD-1 agents. Notably, all anti-PD-1 hypophysitis patients were alive at the time of our analysis. There does not appear to be a clear indication for the routine use of high-dose glucocorticoids in hypophysitis associated with PD-1 blockade, and such dosages were rarely utilized in our cohort. Prior data from our group suggested that high dosages of glucocorticoids may possibly attenuate the anti-tumor efficacy of ipilimumab (2).

Our study did not include patients treated with cemiplimab, an anti-PD-1 agent recently approved by the FDA in September 2018 after the end of our study time period. The frequency of patient follow-up, laboratory testing and MRIs varied due to the retrospective nature of the study. GH stimulation testing was not performed in the patients with hypophysitis since treatment with $\mathrm{GH}$ was contraindicated in these patients due to their underlying malignancy. Central hypothyroidism was also potentially not recognized in a small number of patients with thyroiditis/thyrotoxicosis closely preceding the diagnosis of hypophysitis. Some recent prospective studies with a smaller number of patients have reported slightly higher rates of hypophysitis following treatment with nivolumab or pembrolizumab $(5,6)$. Our medical record database query was restricted to patients who had received a formal endocrinology consultation; we feel that our search criteria were inclusive and likely identified nearly all relevant patients. Comparison with a compilation of prior case reports served as a form of external validation of our findings.

In summary, the present study describes the first cohort of patients diagnosed with hypophysitis following treatment with anti-PD-1 agents, provides a robust risk estimate and establishes a unique phenotype compared to hypophysitis following treatment with ipilimumab or combination therapy with ipilimumab plus nivolumab therapy. Due to the expanding treatment indications for agents targeting PD-1, we anticipate that endocrinologists 
and other practitioners will encounter an increasing number of patients with this treatment complication. Recognition and timely diagnosis of these patients would avoid unnecessary potential effects on morbidity and mortality from untreated adrenal insufficiency.

\section{Declaration of interest}

J V Cohen serves on an advisory board for Sanofi-Genzyme. R J Sullivan serves on advisory boards for Amgen, Array Biopharma, Compugen, Merck, Replimmune, Genentech, and Novartis and receives research support from Merck and Amgen. The other authors have nothing to disclose.

\section{Funding}

This research did not receive any specific grant from any funding agency in the public, commercial or not-for-profit sector.

\section{References}

1 Faje A. Immunotherapy and hypophysitis: clinical presentation, treatment, and biologic insights. Pituitary 201619 82-92. (https:// doi.org/10.1007/s11102-015-0671-4)

2 Faje AT, Lawrence D, Flaherty K, Freedman C, Fadden R, Rubin K, Cohen J \& Sullivan RJ. High-dose glucocorticoids for the treatment of ipilimumab-induced hypophysitis is associated with reduced survival in patients with melanoma. Cancer $20181243706-3714$. (https://doi.org/10.1002/cncr.31629)

3 Faje AT, Sullivan R, Lawrence D, Tritos NA, Fadden R, Klibanski A \& Nachtigall L. Ipilimumab-induced hypophysitis: a detailed longitudinal analysis in a large cohort of patients with metastatic melanoma. Journal of Clinical Endocrinology and Metabolism 201499 4078-4085. (https://doi.org/10.1210/jc.2014-2306)

4 Barroso-Sousa R, Barry WT, Garrido-Castro AC, Hodi FS, Min L, Krop IE \& Tolaney SM. Incidence of endocrine dysfunction following the use of different immune checkpoint inhibitor regimens: a systematic review and meta-analysis. JAMA Oncology 20184 173-182. (https://doi.org/10.1001/jamaoncol.2017.3064)

5 Eggermont AMM, Blank CU, Mandala M, Long GV, Atkinson V, Dalle S, Haydon A, Lichinitser M, Khattak A, Carlino MS et al. Adjuvant pembrolizumab versus placebo in resected stage III melanoma. New England Journal of Medicine 2018378 1789-1801. (https://doi.org/10.1056/NEJMoa1802357)

6 Weber J, Mandala M, Del Vecchio M, Gogas HJ, Arance AM, Cowey CL, Dalle S, Schenker M, Chiarion-Sileni V, Marquez-Rodas I et al. Adjuvant nivolumab versus ipilimumab in resected stage III or IV melanoma. New England Journal of Medicine 2017377 1824-1835. (https://doi.org/10.1056/NEJMoa1709030)

7 Ariyasu R, Horiike A, Yoshizawa T, Dotsu Y, Koyama J, Saiki M, Sonoda T, Nishikawa S, Kitazono S, Yanagitani N et al. Adrenal insufficiency related to anti-programmed death-1 therapy. Anticancer Research 201737 4229-4232. (https://doi.org/10.21873/ anticanres.11814)

8 Boudjemaa A, Rousseau-Bussac G \& Monnet I. Late-onset adrenal insufficiency more than 1 year after stopping pembrolizumab. Journal of Thoracic Oncology 201813 e39-e40. (https://doi.org/10.1016/j. jtho.2017.10.023)

9 Cho KY, Miyoshi H, Nakamura A, Kurita T \& Atsumi T. Hyponatremia can be a powerful predictor of the development of isolated ACTH deficiency associated with nivolumab treatment (Letter to the Editor). Endocrine Journal 201764 235-236. (https:// doi.org/10.1507/endocrj.EJ16-0596)
10 Fujimura T, Kambayashi Y, Furudate S, Kakizaki A, Hidaka T, Haga T, Hashimoto A, Morimoto R \&Aiba S. Isolated adrenocorticotropic hormone deficiency possibly caused by nivolumab in a metastatic melanoma patient. Journal of Dermatology 201744 e13-e14. (https:// doi.org/10.1111/1346-8138.13532)

11 Ishikawa $\mathrm{M} \&$ Oashi K. Case of hypophysitis caused by nivolumab. Journal of Dermatology $2017 \mathbf{4 4}$ 109-110. (https://doi. org/10.1111/1346-8138.13437)

12 Kastrisiou M, Kostadima FL, Kefas A, Zarkavelis G, Kapodistrias N, Ntouvelis E, Petrakis D, Papadaki A, Vassou A \& Pentheroudakis G. Nivolumab-induced hypothyroidism and selective pituitary insufficiency in a patient with lung adenocarcinoma: a case report and review of the literature. ESMO Open 20172 e000217. (https:// doi.org/10.1136/esmoopen-2017-000217)

13 Kitajima K, Ashida K, Wada N, Suetsugu R, Takeichi Y, Sakamoto S, Uchi H, Matsushima T, Shiratsuchi M, Ohnaka K et al. Isolated ACTH deficiency probably induced by autoimmune-related mechanism evoked with nivolumab. Japanese Journal of Clinical Oncology 201747 463-466. (https://doi.org/10.1093/jico/hyx018)

14 Kitano S, Tatsuno K, Ishibe J, Shimauchi T, Fujiyama T, Ito T, Ogawa N \& Tokura Y. Isolated adrenocorticotropic hormone deficiency in melanoma patients treated with nivolumab. Acta Dermato-Venereologica 201898 704-705. (https://doi. org/10.2340/00015555-2902)

15 Kuru S, Khan N \& Shaaban H. Acute hypophysitis secondary to nivolumab immunotherapy in a patient with metastatic melanoma. International Journal of Critical Illness and Injury Science $2017 \mathbf{7}$ 177-180. (https://doi.org/10.4103/IJCIIS.IJCIIS_15_17)

16 Marchand L, Paulus V, Fabien N, Perol M, Thivolet C, Vouillarmet J $\&$ Saintigny P. Nivolumab-induced acute diabetes mellitus and hypophysitis in a patient with advanced pulmonary pleomorphic carcinoma with a prolonged tumor response. Journal of Thoracic Oncology 201712 e182-e184. (https://doi.org/10.1016/j. jtho.2017.07.021)

17 Mishima Y, Fukaishi T, Inase N \& Isogai S. Nivolumabinduced hypophysitis, secondary adrenal insufficiency and destructive thyroiditis in a patient with lung adenocarcinoma. Internal Medicine 201958 693-697. (https://doi.org/10.2169/ internalmedicine.1268-18)

18 Narahira A, Yanagi T, Cho KY, Nakamura A, Miyoshi H, Hata H, Imafuku K, Kitamura S \& Shimizu H. Isolated adrenocorticotropic hormone deficiency associated with nivolumab therapy. Journal of Dermatology 201744 e70. (https://doi.org/10.1111/1346-8138.13571)

19 Oda T, Sawada Y, Okada E, Yamaguchi T, Ohmori S, Haruyama S, Yoshioka M \& Nakamura M. Hypopituitarism and hypothyroidism following atrioventricular block during nivolumab treatment. Journal of Dermatology 201744 e144-e145. (https://doi.org/10.1111/13468138.13797)

20 Ohara N, Ohashi K, Fujisaki T, Oda C, Ikeda Y, Yoneoka Y, Hashimoto T, Hasegawa G, Suzuki K \& Takada T. Isolated adrenocorticotropin deficiency due to nivolumab-induced hypophysitis in a patient with advanced lung adenocarcinoma: a case report and literature review. Internal Medicine 201857 527-535. (https://doi.org/10.2169/internalmedicine.9074-17)

21 Okano Y, Satoh T, Horiguchi K, Toyoda M, Osaki A, Matsumoto S, Tomaru T, Nakajima Y, Ishii S, Ozawa A et al. Nivolumab-induced hypophysitis in a patient with advanced malignant melanoma. Endocrine Journal 201663 905-912. (https://doi.org/10.1507/endocrj. EJ16-0161)

22 Otsubo K, Nakatomi K, Furukawa R, Ashida K, Yoneshima Y, Nakanishi Y \& Okamoto I. Two cases of late-onset secondary adrenal insufficiency after discontinuation of nivolumab. Annals of Oncology 201728 3106-3107. (https://doi.org/10.1093/annonc/mdx497)

23 Seki T, Yasuda A, Oki M, Kitajima N, Takagi A, Nakajima N, Miyajima A \& Fukagawa M. Secondary adrenal insufficiency following nivolumab therapy in a patient with metastatic renal cell 
carcinoma. Tokai Journal of Experimental and Clinical Medicine 201742 115-120.

24 Shrotriya S, Rai MP, Alratroot A \& Sarzynski E. Delayed presentation of isolated adrenocorticotropin insufficiency after nivolumab therapy for advanced non-small-cell lung carcinoma (NSCLC). BMJ Case Reports 20182018 bcr-2018-225048. (https://doi.org/10.1136/bcr2018-225048)

25 Takaya K, Sonoda M, Fuchigami A \& Hiyoshi T. Isolated adrenocorticotropic hormone deficiency caused by nivolumab in a patient with metastatic lung cancer. Internal Medicine 201756 2463-2469. (https://doi.org/10.2169/internalmedicine.8548-16)

26 Takebayashi K, Ujiie A, Kubo M, Furukawa S, Yamauchi M, Shinozaki H, Suzuki T, Naruse R, Hara K, Tsuchiya T et al. Isolated adrenocorticotropic hormone deficiency and severe hypercalcemia after destructive thyroiditis in a patient on nivolumab therapy with a malignant melanoma. Journal of Clinical Medicine Research 201810 358-362. (https://doi.org/10.14740/jocmr3257w)

27 Tsukizawa Y, Kondo K, Ichiba T, Naito H, Mizuki K \& Masuda K. Refractory hypotension due to Nivolumab-induced adrenal insufficiency. Nagoya Journal of Medical Science 201880 285-288.

28 Zeng MF, Chen LL, Ye HY, Gong W, Zhou LN, Li YM \& Zhao XL. Primary hypothyroidism and isolated ACTH deficiency induced by nivolumab therapy. Medicine 201796 e8426. (https://doi. org/10.1097/MD.0000000000008426)

29 Neril R, Lorton J \& Zonszein J. Nivolumab-induced isolated adrenal insufficiency: a case report. AACE Clinical Case Reports 20173 e210-e212. (https://doi.org/10.4158/EP161491.CR)

30 von Elm E, Altmen DG, Egger M, Pocock SJ, Gotzsche PC, Vandenbroucke JP \& STROBE Initiative. The strengthening the reporting of observational studies in epidemiology (STROBE) statement: guidelines for reporting observational studies. PLoS Medicine 20074 e296. (https://doi.org/10.1371/journal. pmed.0040296)

31 Faje AT \& Sullivan RJ. Reply to emergency management of immunerelated hypophysitis: collaboration between specialists is essential to achieve optimal outcomes. Cancer 2018124 4732. (https://doi. org/10.1002/cncr.31786)

32 Byun DJ, Wolchok JD, Rosenberg LM \& Girotra M. Cancer immunotherapy - immune checkpoint blockade and associated endocrinopathies. Nature Reviews Endocrinology 201713 195-207. (https://doi.org/10.1038/nrendo.2016.205)

33 Su Q, Zhang XC, Wang DY, Zhang HR, Zhu C, Hou YL, Liu JL \& Gao ZH. The risk of immune-related endocrine disorders associated with anti-PD-1 inhibitors therapy for solid tumors: a systematic review and meta-analysis. International Immunopharmacology 201859 328-338. (https://doi.org/10.1016/j.intimp.2018.04.021)

34 Hodi FS, O'Day SJ, McDermott DF, Weber RW, Sosman JA, Haanen JB, Gonzalez R, Robert C, Schadendorf D, Hassel JC et al. Improved survival with ipilimumab in patients with metastatic melanoma. New England Journal of Medicine 2010363 711-723. (https://doi. org/10.1056/NEJMoa1003466)

35 Postow MA, Chesney J, Pavlick AC, Robert C, Grossmann K, McDermott D, Linette GP, Meyer N, Giguere JK, Agarwala SS et al. Nivolumab and ipilimumab versus ipilimumab in untreated melanoma. New England Journal of Medicine 2015372 2006-2017. (https://doi.org/10.1056/NEJMoa1414428)

36 Caturegli P, Di Dalmazi G, Lombardi M, Grosso F, Larman HB, Larman T, Taverna G, Cosottini M \& Lupi I. Hypophysitis secondary to cytotoxic T-lymphocyte-associated protein 4 blockade: insights into pathogenesis from an autopsy series. American Journal of Pathology 2016186 3225-3235. (https://doi.org/10.1016/j. ajpath.2016.08.020)

37 Iwama S, De Remigis A, Callahan MK, Slovin SF, Wolchok JD \& Caturegli P. Pituitary expression of CTLA-4 mediates hypophysitis secondary to administration of CTLA-4 blocking antibody. Science Translational Medicine 20146 230ra45. (https://doi.org/10.1126/ scitranslmed.3008002)

38 Dougan M. Checkpoint blockade toxicity and immune homeostasis in the gastrointestinal tract. Frontiers in Immunology 201781547. (https://doi.org/10.3389/fimmu.2017.01547)

Received 30 March 2019

Revised version received 29 May 2019

Accepted 6 June 2019 